\title{
A Genome-Wide Association Study of Field Resistance to Magnaporthe Oryzae in Rice
}

Dan Zhu ${ }^{1,2+}$, Houxiang Kang ${ }^{1 \dagger}$, Zhiqiang Li ${ }^{1 \dagger}$, Minghao Liu' ${ }^{1,2}$, Xiaoli Zhu', Yue Wang ${ }^{2}$, Dan Wang ${ }^{2}$, Zhilong Wang ${ }^{2}$, Wende Liu' ${ }^{1}$ and Guo-Liang Wang ${ }^{1,2,3^{*}}$ (1)

\begin{abstract}
Background: Breeding of rice cultivars with long-lasting resistance to the rice blast fungus Magnaporthe oryzae is difficult, and identification of new resistance genes is essential. Most of the loci associated with blast resistance against M. oryzae in rice have been identified in controlled environments and with single isolates, and such loci may confer resistance to only a small faction of the $M$. oryzae strains. In the field, however, rice is commonly attacked by multiple strains. Research is therefore needed to identify loci that confer resistance in the field, i.e., "field blast resistance". To identify loci associated with field blast resistance (LAFBRs), we conducted a genome-wide association study (GWAS) using the rice diversity panel 1 (RDP1) cultivars. These cultivars were evaluated in the field in three major rice production areas of China.
\end{abstract}

Results: GWAS identified 16 LAFBRs. Among them, 13 are novel and the other three are co-localized with known blast resistance regions. Seventy-four candidate genes are identified in the 16 LAFBR regions, which encode receptor-like protein kinases, transcription factors, and other defense-related proteins. Using the rice transcriptome data, compared with the rice-rice blast compatible interaction, we identified seven candidate genes that are significantly up-regulated and five genes that are significantly down-regulated in the incompatible interaction among the candidate genes.

Conclusions: We identified 16 LAFBRs involved in field resistance to M. oryzae and 20 cultivars that exhibit high levels of resistance in both the field and growth chamber. The resistant cultivars and the SNP markers identified in this study should be useful for marker-assisted selection of new rice cultivars that confer high levels of resistance against $M$. oryzae field populations.

Keywords: Rice, Magnaporthe oryzae, GWAS, Field blast resistance, LAFBR

\section{Background}

Rice (Oryzae sativa L.) is an important food crop that feeds more than half of the world's population (Khush 2005). Rice blast, caused by the fungal pathogen Magnaporthe oryzae, is a destructive disease of rice that reduces yields from 10 to $30 \%$ annually (Skamnioti and Gurr 2009). The most effective and economical way to control the disease is via resistant cultivars (Hulbert et al., 2003). To date, more than

\footnotetext{
* Correspondence: wang.620@osu.edu

Dan Zhu, Houxiang Kang and Zhiqiang Li are co-first authors of the paper. ${ }^{\dagger}$ Equal contributors

'State Key Laboratory for Biology of Plant Diseases and Insect Pests, Institute of Plant Protection, Chinese Academy of Agricultural Sciences, Beijing 100193, People's Republic of China

${ }^{2}$ Hunan Provincial Key Laboratory of Crop Germplasm Innovation and Utilization and College of Agronomy, Hunan Agricultural University,

Changsha, Hunan 410128, People's Republic of China

Full list of author information is available at the end of the article
}

100 blast resistance (R) genes and about 500 quantitative trait loci (QTLs) have been identified (Ashkani et al., 2015), and 25 of them have been cloned (Wu et al., 2015; Zheng et al. 2016). However, rice cultivars often lose their resistance to $M$. oryzae within 3-5 years because of the high variability of the fungus in the field (Oliveira-Garcia and Valent 2015; Devi et al. 2015). In major production areas in China, for example, 174 resistant rice cultivars (disease index $<4$ on a scale from 0 to 9) released from 2004 to 2008 lost their blast resistance (Feng et al. 2014). It is therefore necessary to identify new rice blast $\mathrm{R}$ genes that will be effective against M. oryzae field populations for extended periods.

The classic genetic linkage mapping strategy using biparental crosses has been widely used to identify $R$ genes and QTLs. Because this strategy requires the construction of a mapping population and genotyping, however, it is labor intensive and time consuming. The genome- 
wide association study (GWAS) method based on the high-density SNP markers has recently been established in plants such as maize (Yu et al. 2006), rice (Huang et al. 2010 and Zhao et al. 2011), and soybean (Hwang et al. 2014). Compared with the traditional bi-parental mapping strategy, the genetic background of the population for GWAS is much more diverse, which can be used for mapping of rare alleles of agronomic traits. Recently, in rice, dozens of new genes/QTLs associated with different phenotypes have been identified with GWAS because the method efficiently dissects the genetic structure of complicated phenotypes (Zhao et al. 2011; Spindel et al. 2016). The rice diversity panel 1 (RDP1), which consists of over 400 O. sativa cultivars collected from 82 countries, is publically available and contains substantial genetic and phenotypic diversity (Eizenga et al. 2014; Ali et al. 2010; Eizenga et al. 2013). Importantly, a high-density SNP map for the RDP1 has been generated and is publically available. Researchers have used the RDP1 to identify many genes/QTLs associated with important agronomic traits in rice (Zhao et al. 2011; Norton et al. 2014; Copenhaver et al. 2011). Using the same RDP1 and growth chamber assays, Kang et al. (2016) recently identified 97 loci associated with blast resistance (LABRs) against five $M$. oryzae isolates.

In this study, we evaluated the resistance of the RDP1 cultivars in rice blast nurseries (field sites containing highly diverse $M$. oryzae populations) in three representative rice production regions of China. Association mapping showed that 16 loci associated with field blast resistance (LAFBRs) are significantly linked to rice blast field resistance. The resistant cultivars and the LAFBRs identified in this study will be useful for the breeding of blast resistance in rice.

\section{Results}

\section{Evaluation of the Field Blast Resistance of the RDP1 Cultivars}

A total of 373, 356, and 336 rice cultivars in the RDP1 were grown in the blast nurseries of the three Chinese rice production areas: Shanghang (in Southeast China, Fujian Province), Wuchang (in Northeast China, Heilongjiang Province), and Taojiang (in Central China, Hunan Province) (Fig. 1a-c; Additional file 1: Table S1). Consistent with previous results obtained under growth chamber conditions (Kang et al. 2016), a large range of resistant phenotypes in the RDP1 were detected in the three nurseries. At Wuchang, $61.6 \%$ (207 of 336) of the cultivars were resistant (with a score of 0 to 3 on a disease severity scale from 0 to 9) (Fig. 1d), compared to $46.9 \%$ (175 of 373) at Shanghang and $27.8 \%$ (99 of 356) at Taojiang (Fig. 1e, f). These results indicated that the blast disease pressure was highest at the Taojiang site.
To understand the differences in resistance among the cultivars at the three sites, we performed pair-wise comparisons of cultivars' disease reactions. The analysis showed that $70.9 \%$ (107 of 151) of the resistant cultivars at Shanghang were also resistant at Wuchang. In contrast, only $51.7 \%$ (107 of 207) of the resistant cultivars at Wuchang were resistant at Shanghang (Fig. 1g). Of the resistant cultivars at Taojiang, $61.6 \%$ (61 of 99) were also resistant at Shanghang. In contrast, only $37.0 \%$ (61 of 165) of the resistant cultivars at Shanghang were also resistant at Taojiang (Fig. 1h). A high percentage of resistant cultivars $(67.3 \%, 66$ out of 98) at Taojiang were also resistant at Wuchang but only $31.9 \%$ (66 of 207) of the resistant cultivars at Wuchang were resistant at Taojiang (Fig. 1i). These results suggest that the RDP1 cultivars that are highly resistant to the $M$. oryzae population at Taojiang have broad-spectrum resistance. Further analysis revealed that 40 cultivars were resistant to the $M$. oryzae populations at all three locations (Additional file 2: Table S2). Of these 40 cultivars, 20 were also resistant to the five $M$. oryzae isolates collected from five countries (Kang et al. 2016). Information on these 20 cultivars is provided in Table 1.

Based on the evaluation of disease at the three field sites, we analyzed the differences in rice blast resistance among sub-populations of the RDP1. First, we constructed the phylogenetic tree of the RDP1 cultivars using 3835 high quality SNP markers selected from the 44-K SNP dataset (Zhao et al. 2011). The analysis showed that the RDP1 has five major sub-populations (indica [IND], aus [AUS], tropical japonica [TRJ], temperate japonica [TEJ], and aromatic [ARO]) and an additional sub-population (admixture [ADM]). The genetic distance between TRJ and TEJ is very small (Fig. 2a). When the blast scores were classified according to the sub-populations, we found two main features. First, the ratio of resistant to susceptible cultivars in AUS and IND sub-populations was close to 0.5 in Central and Southeastern China (Taojiang and Shanghang) but was greater than 0.5 in the two sub-populations in Northeastern China (Wuchang). Second, in all three areas, more than half of the cultivars $(68.6,51.3$, and $82.4 \%$ in Shanghang, Taojiang, and Wuchang, respectively) in the TRJ sub-population were resistant, and most of the cultivars (84.4, 89.4, and $83.9 \%$ in Shanghang, Taojiang, and Wuchang, respectively) in the TEJ sub-population were susceptible (Fig. 2b-d). Although the genetic distance between TRJ and TEJ sub-populations is less than that between the other sub-populations (Fig. 2a), blast resistance is higher in TRJ than TEJ.

\section{Identification of LAFBRs}

Using the 44-K SNP data set and the disease resistance scores of the cultivars, we identified 16 non-redundant 
LAFBRs (Table 2). Among these loci, eight (LAFBR_1, 2, $8,9,12,14,15$, and 16) were associated with the resistance to the $M$. oryzae population in Shanghang, five (LAFBR_3, 4, 7, 11, and 12) were associated with the resistance to the $M$. oryzae population in Wuchang, and only one (LAFBR_5) was associated with the resistance to the $M$. oryzae population in Taojiang. Two loci (LAFBR_6 and 10) were associated with the resistance to the $M$. oryzae population in both Wuchang and
Shanghang (Fig. 3a-c). The 16 LAFBRs are located on chromosome $1,3,4,5,8,9,11$, and 12 in the rice genome.

Next, we identified 74 candidate genes in the LAFBR regions and obtained their annotation information (Additional file 3: Table S3). None of them are homologous to any known NBS-type R gene family in the rice genome (Liu et al. 2015; Liebranda et al. 2013; Hu et al. 2005) (Additional file 3: Table S3). We classified those candidate

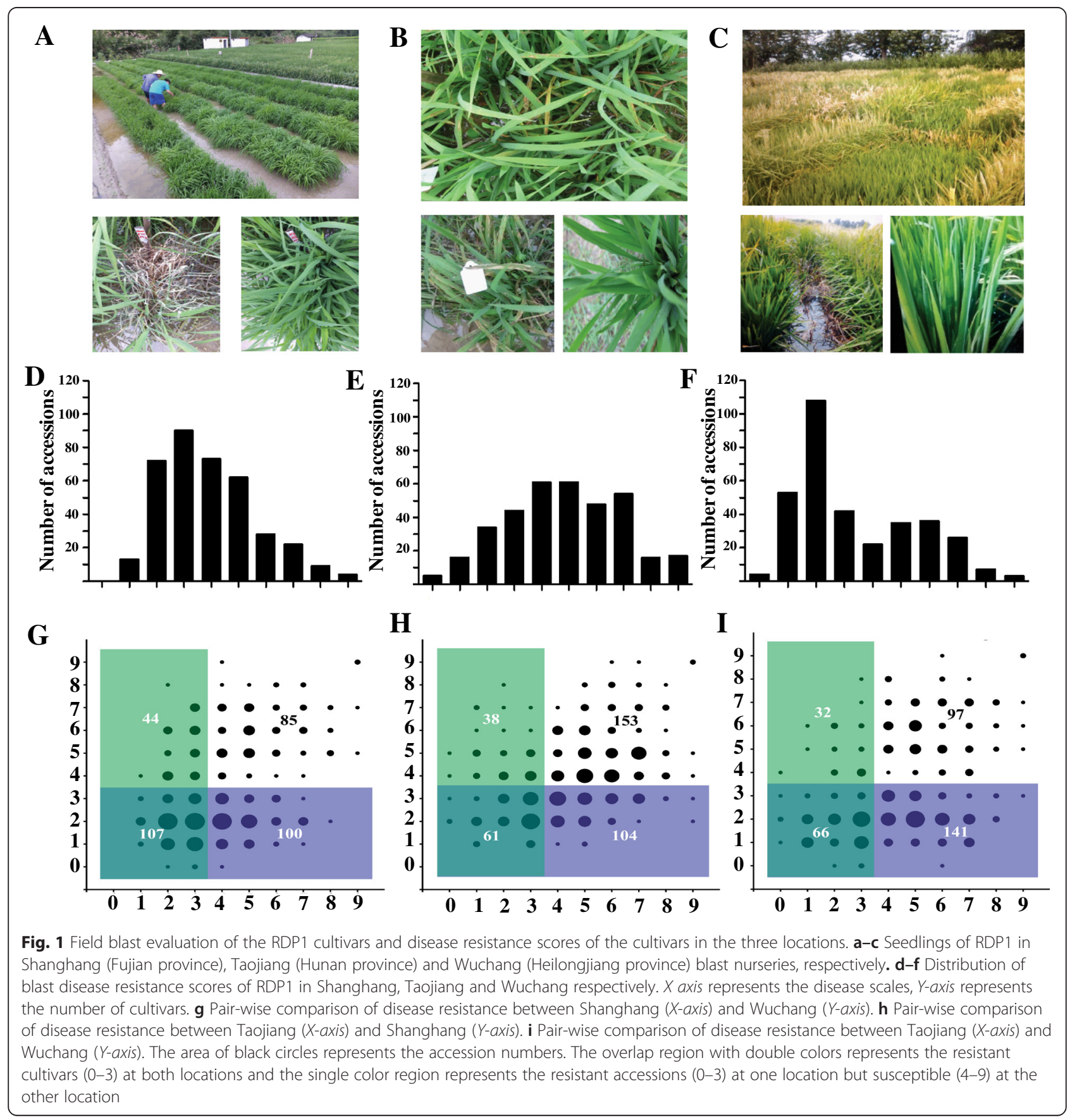


Table 1 Information concerning the 20 cultivars of rice that were highly resistant to rice blast disease under both field and growth chamber conditions

\begin{tabular}{|c|c|c|c|c|c|}
\hline Accession or name & GSOR ID & IRGC ID & NSFTV. ID & Sub-population & Origin \\
\hline Dom-sufid & 301042 & 117721 & 45 & ARO & Iran \\
\hline Geumobyeo & 301052 & 117612 & 56 & TEJ & South Korea \\
\hline IRAT 177 & 301066 & 117761 & 73 & TRJ & French Guiana \\
\hline Jambu & 301068 & 117764 & 75 & TRJ & Indonesia \\
\hline LAC 23 & 301091 & 117796 & 99 & TRJ & Liberia \\
\hline NSF-TV 116 & 301108 & 117824 & 116 & TRJ & Pakistan \\
\hline B6616A4-22-Bk-5-4 & 301158 & 117646 & 167 & TRJ & United States \\
\hline Zhenshan 2 & 301163 & 117944 & 172 & IND & China \\
\hline IRAT 13 & 301186 & 117760 & 195 & TRJ & Cote D'Ivoire \\
\hline WAB 502-13-4-1 & 301229 & 117932 & 239 & TRJ & Cote D'Ivoire \\
\hline SL 22-613 & 301284 & 117756 & 294 & ADM & Sierra Leone \\
\hline Llanero 501 & 301298 & 117805 & 308 & TRJ & Venezuela \\
\hline Manzano & 301299 & To be assigned & 309 & TRJ & Zaire \\
\hline R 101 & 301300 & 117857 & 310 & TRJ & Zaire \\
\hline DNJ 140 & 301313 & 117718 & 323 & AUS & Bangladesh \\
\hline Berenj & 301330 & 117656 & 340 & ADM & Afghanistan \\
\hline OS 6 (WC 10296) & 301378 & 117830 & 395 & TRJ & Zaire \\
\hline Cocodrie & 301379 & 117693 & 396 & TRJ & United States \\
\hline Saber & 301411 & To be assigned & 630 & TRJ & United States \\
\hline C101A51 & 301420 & To be assigned & 626 & IND & Colombia \\
\hline
\end{tabular}

GSOR ID Genetic Stocks-Oryza collection identification number, IRGC ID International Rice Germplasm Collection identification number

genes into nine gene families based on the gene annotation information: 1) $20.3 \%$ (15 of 74) belong to the receptor-like protein kinase gene family; 2) $16.2 \%$ (12 of 74) are transcription factor genes; 3) $12.2 \%$ (9 of 74) are ubiquitin-related genes; 4) $12.2 \%$ (9 of 74) are phosphorylation-related genes; 5 ) $10.8 \%$ (8 of 74) are DNA/ATP-binding genes; 6) $8.1 \%$ (6 of 74) are oxidase/oxidoreductase genes; 7) $5.4 \%$ (4 of 74) are heat shock protein genes; 8) $4.1 \%$ (3 of 74) are LRR type genes; and 9) $10.8 \%$ (8 of 74) are other defence related genes (Fig. 4).

To measure the expression of the candidate genes during rice-blast compatible and incompatible interactions, we analyzed the RNA-seq transcriptome data sets (Kawahara et al. 2012). When compared with the rice-blast compatible interaction, among the candidate genes, $9.5 \%$ (7 of 74) were up-regulated and $6.8 \%$ (5 of 74) were down-regulated during the incompatible interaction (Additional file 4: Table S4). These 12 genes, whose expression patterns differed between the compatible and incompatible rice-blast interactions, are the strong candidate genes that are associated with field blast resistance.
Comparison of Phenotypic and GWAS Results Obtained in the Field Blast Nurseries vs. Growth Chambers

To understand rice blast resistance under natural and artificial conditions, we compared the blast evaluations and GWAS results obtained in the three fields in this study with those obtained in the growth chambers in a previous study (Kang et al. 2016). Based on all of the RDP1 cultivars, pair-wise correlation analysis suggested that the resistance level in the field was positively correlated with the resistance level in the growth chamber. The correlation, however, was quite low ( $r$ values of 0.06 and 0.32 ) in two cases. The correlation between $\mathrm{Wu}$ chang field data and RB22 single-isolate inoculation in a growth chamber is the highest $(0.32, P<0.05)$ (Additional file 5: Table S5). These results suggest that, although the resistance of a few cultivars is similar, the resistance of most cultivars is different in the growth chamber and in the field.

We then compared the 16 LAFBRs obtained in this study with the 97 LABRs previously identified using the RDP1 (Kang et al. 2016) and found that only three LAFBRs are co-localized with LABRs: LAFBR_7 and LABR_44; LAFBR_9 and LABR_55; and LAFBR_11 and 
A

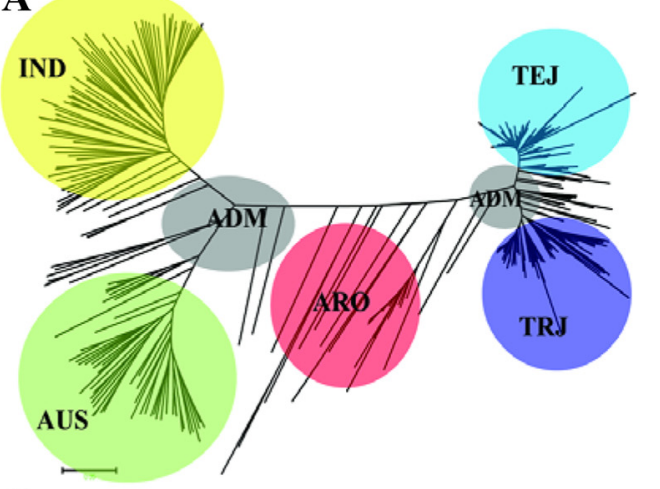

C

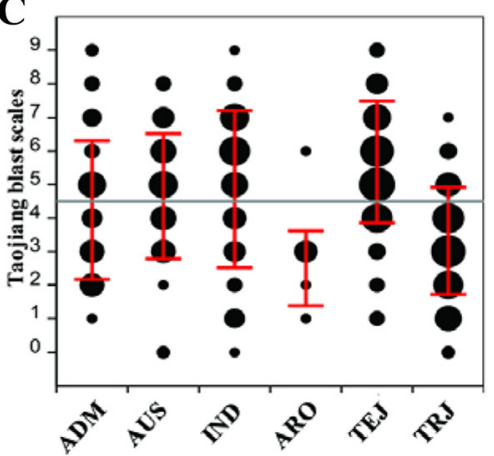

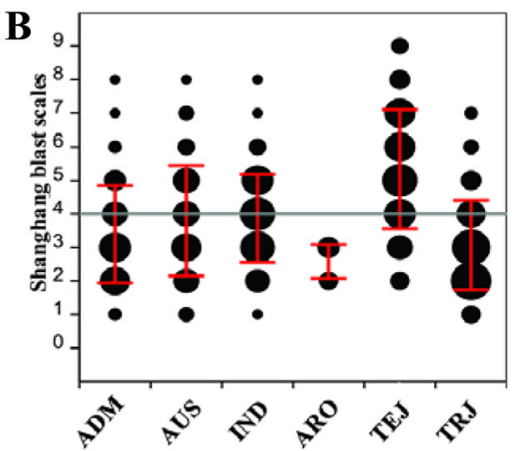

D

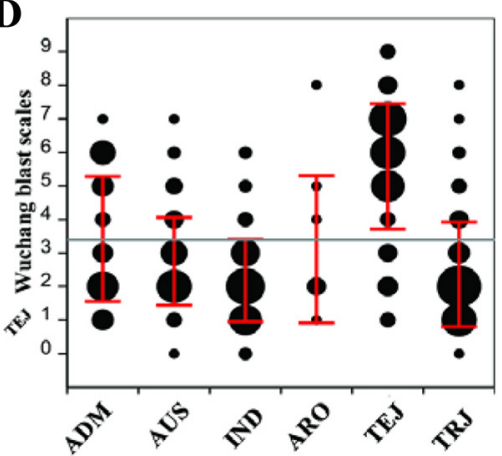

Fig. 2 Grouping of the RDP1 cultivars and blast resistance of the different sub-populations. a Phylogenetic tree of RDP1. ADM =admixture; $\mathrm{ARO}=$ aromatic; $\mathrm{AUS}=$ aus; IND = indica; TEJ = temperate japonica; TRJ = tropic japonica. Distribution of blast resistance scores of RDP1 in the six sub-populations in Shanghang (b), Taojiang (c) and Wuchang $(\mathbf{d})$, respectively. The area of black circles represent the accession numbers, the red line represents the standard deviation (SD) of the blast resistance scores in each sub-population, and the gray line represents the average level of the blast resistance scores of the RDP1 cultivars

Table 2 The LAFBRs that are associated with the field resistance in the three field blast nurseries

\begin{tabular}{|c|c|c|c|c|c|c|c|}
\hline LAFBRs & Chr. & Positions & Top SNP & Top SNP genotypes & $\mathrm{R}$ associated SNP & MAF (\%) & Locus reference \\
\hline LAFBR_1 & 1 & $25768623-26002852$ & id1015310 & $C / T$ & $\mathrm{~T}$ & 32.51 & \\
\hline LAFBR_2 & 1 & $26034806-26256424$ & id1015389 & $\mathrm{G} / \mathrm{T}$ & T & 31.29 & \\
\hline LAFBR_3 & 1 & $28473508-28602492$ & id1016715 & $A / T$ & A & 29.90 & \\
\hline LAFBR_4 & 1 & 29430517-29612599 & id1017391 & $\mathrm{A} / \mathrm{G}$ & G & 31.84 & \\
\hline LAFBR_5 & 1 & $32130307-32194257$ & id1027545 & $\mathrm{G} / \mathrm{T}$ & T & 14.47 & \\
\hline LAFBR_6 & 3 & $11173192-11364668$ & id3005883 & $\mathrm{G} / \mathrm{T}$ & G & 27.91 & \\
\hline LAFBR_7 & 4 & $31260971-31367925$ & id4010692 & $C / G$ & C & 30.83 & LABR_44 \\
\hline LAFBR_8 & 5 & $2450054-2703130$ & id5001423 & $\mathrm{A} / \mathrm{C}$ & C & 27.43 & \\
\hline LAFBR_9 & 8 & $5278331-5384087$ & id8001749 & $A / G$ & G & 30.83 & LABR_55 \\
\hline LAFBR_10 & 8 & 21688770-21871571 & id8006180 & $A / G$ & G & 25.93 & \\
\hline LAFBR_11 & 9 & 16850040-17094483 & id9005401 & $\mathrm{G} / \mathrm{T}$ & $\mathrm{T}$ & 33.01 & LABR_71 \\
\hline LAFBR_12 & 10 & $16875502-17129435$ & id10004848 & $C / G$ & G & 24.47 & \\
\hline LAFBR_13 & 10 & $19737153-19958786$ & id10006307 & $\mathrm{A} / \mathrm{C}$ & C & 23.50 & \\
\hline LAFBR_14 & 11 & $4415292-4486716$ & id1 1001788 & $A / G$ & G & 29.78 & \\
\hline LAFBR_15 & 11 & $23768147-23957505$ & id1 1009444 & $\mathrm{C} / \mathrm{G}$ & C & 19.55 & \\
\hline LAFBR_16 & 12 & $24903601-25001217$ & id12009011 & $\mathrm{C} / \mathrm{T}$ & C & 28.20 & \\
\hline
\end{tabular}




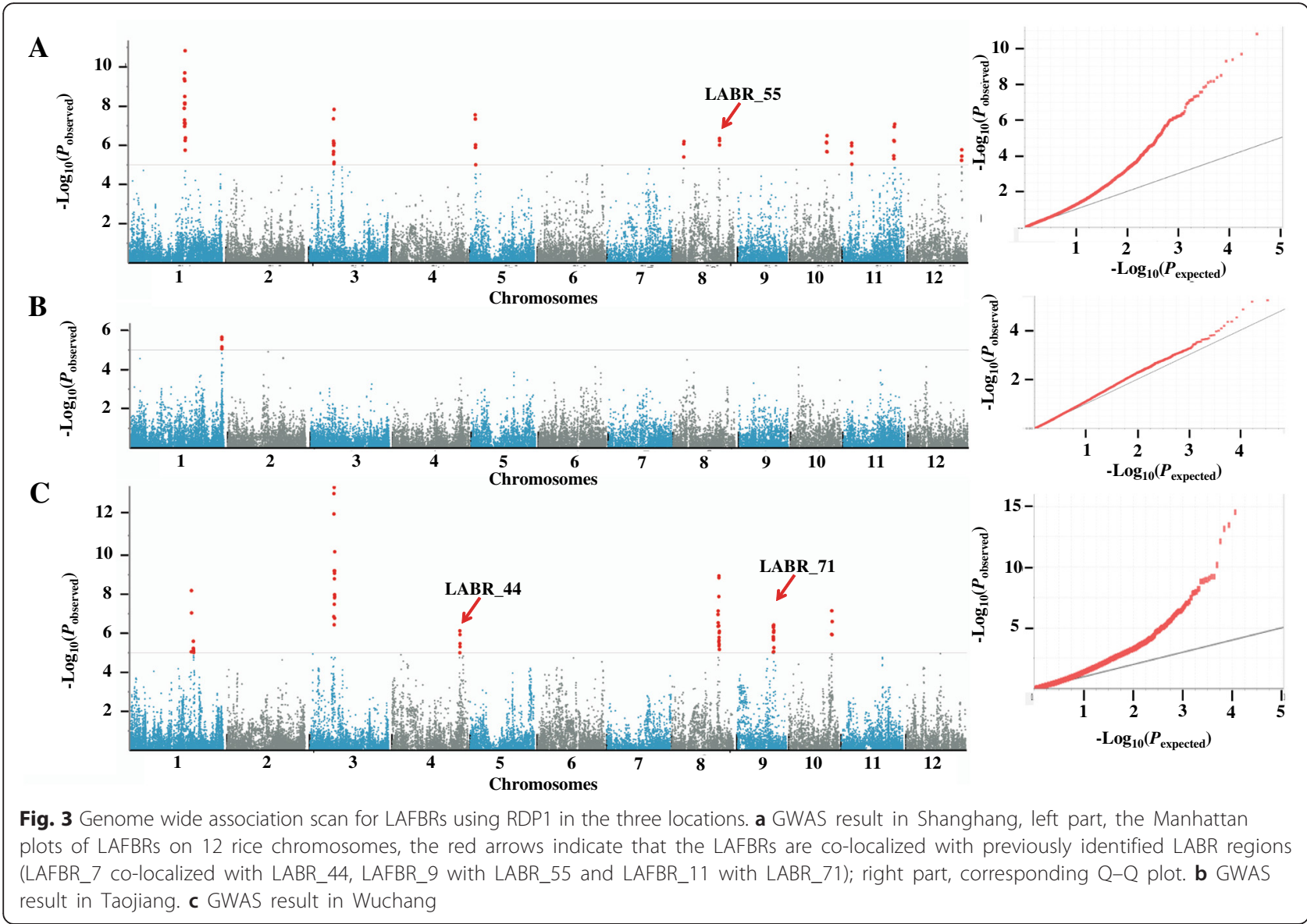

LABR_71. Interestingly, none of the 16 LAFBRs was colocalized with $\mathrm{R}$ genes previously identified using a traditional mapping strategy. We also compared the 16 loci with previously reported $\sim 70$ major rice blast resistance loci including 21 cloned genes (Liu et al., 2014), and didn't find any overlap regions. Therefore, 13 of the LAFBRs identified in this study are considered to be novel.
Genotype Analysis of 20 Highly Resistant Rice Cultivars

We identified 20 rice cultivars that exhibited high levels of resistance both in the field (the current study) and in growth chambers (Kang et al. 2016). These cultivars come from 15 countries and belong to different subpopulations (Table 1), including two cultivars in IND, 13 TRJ, one TEJ, one ARO, two ADM, and one AUS.

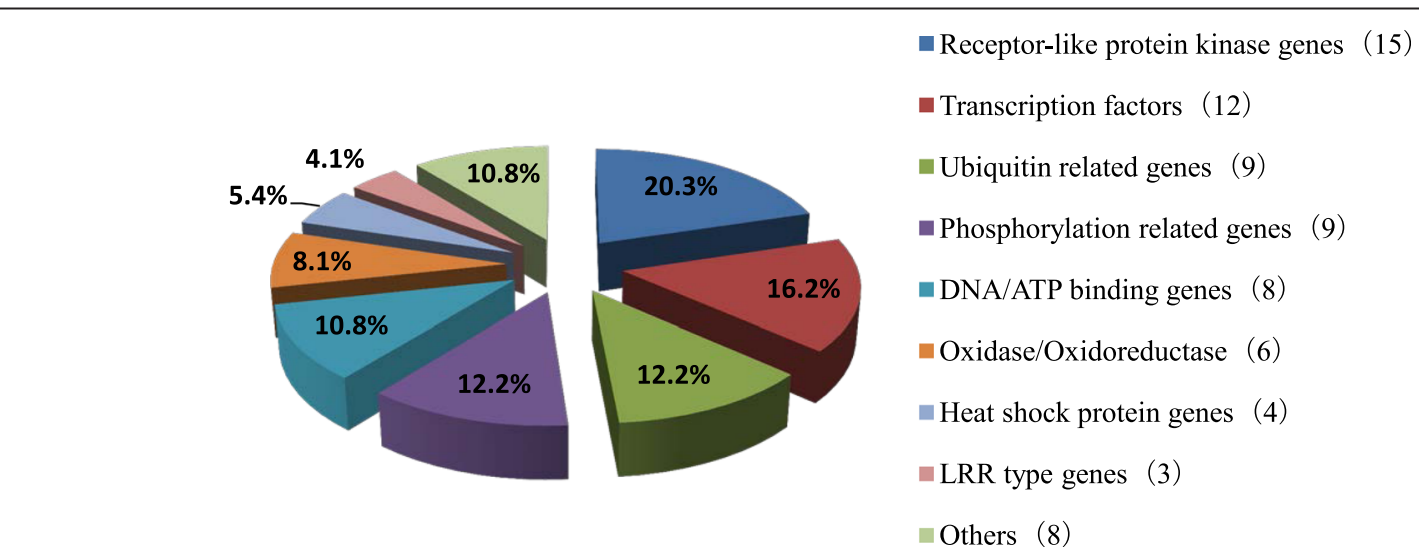

Fig. 4 Classification of the 74 candidate genes in the 16 LAFBRs. We manually classified the candidate genes in the 16 LAFBRs using both the MSU (v7.0) rice gene annotation information 
Interestingly, $65 \%$ (13 of 20) of the highly resistant cultivars are from the TRJ sub-population. To determine the genotype of these rice cultivars, we analyzed the haplotype of the 16 LAFBRs in the cultivars (Additional file 6: Table S6). The analysis showed that the average frequency of the R-type alleles for all 20 cultivars is $89.9 \%$. Among the 20 cultivars, six (301108, 301186, 301229, 301299, 301330, and 301378) contain $100.0 \%$ R-type alleles. These results demonstrate that the $\mathrm{R}$ genotypes are highly enriched in the 20 resistant cultivars and that these cultivars could be valuable resistant-donor materials for rice blast breeding as well as for rice blast gene mapping and cloning.

\section{Discussion}

Understanding the molecular basis of field resistance to rice blast is challenging because of the lack of appropriate mapping populations and of a reliable disease evaluation method in the field. In this study, we evaluated the resistance of the RDP1 cultivars in three blast nurseries located in different geographic/climatic rice production areas in China. We found that $61.6,46.9$, and $27.8 \%$ of the RDP1 cultivars are resistant in Wuchang (in Heilongiiang Province in Northeast China), Shanghang (in Fujian Province in Southeast China), and Taojiang (in Hunan Province in Central China), respectively. Through GWAS, we identified 16 LAFBRs involved in field blast resistance. Among these LAFBRs, 13 are novel and the other three are co-localized with known blast resistance regions. One, five, and eight LAFBRs were associated with the field resistance in Taojiang, Shanghang, and Wuchang, respectively, and two loci (LAFBR_6 and LAFBR_10) were associated with the field blast resistance in both Wuchang and Shanghang. This is the first effort to use GWAS in order to identify loci that confer blast resistance in the field.

NBS-LRR type genes form the largest $\mathrm{R}$ gene families in plants (Dangl and Jones 2001; Meyers et al. 2003; Jones and Dangl 2006). In a previous GWAS that evaluated single isolates in growth chambers, researcher found that a large number of NBS-LRR type R are associated with the resistance to $M$. oryzae (Kang et al. 2016). In the current study, however, we failed to find an NBS-LRR-type gene among the 16 LAFBRs. The main genes found are those encoding receptor-like protein kinases and transcription factors. These results suggest that the NBS-LRR genes identified in the growth chamber may be more effective against single blast strains than against multiple blast strains. Under blast nurseries conditions, in contrast, the specific NBS-LRR R genes are difficult to detect because of the diversity of $M$. oryzae strains. Consequently, the genes involved in the recognition and signaling of the PAMP-triggered immunity, such as receptor-like protein kinase genes and transcription factors are more likely to be activated in the blast nurseries than in the growth chamber, and these genes may play more important roles in blast resistance in the blast nurseries than in the growth chamber (single isolate condition).

A previous study showed that the most susceptible subpopulation (mean disease score $=7.0$ ) in the RDP1 against M. oryzae strains in the USA is TEJ (Ali et al. 2010). Similarly, we found that the TEJ group is the most susceptible sub-population to field blast populations in China, and Kang et al. (2016) found that the TEJ group is the most susceptible sub-population to single rice blast isolates. Ali et al. (2010) found that IND is the most resistant subpopulation (mean disease score $=3.0$ ) against USA strains of $M$. oryzae. However, our blast evaluations under both artificial and field conditions indicated that TRJ is the most resistant sub-population. These results suggest that cultivars in the TRJ sub-population are a valuable resource for the breeding of rice blast resistance.

We found 40 cultivars that are highly resistant in all three of the tested fields in China. Among the 40 cultivars, 20 are also reported to be resistant against all five of the diverse $M$. oyzae isolates under growth chamber conditions (Kang et al. 2016). Among the 20 cultivars, six (301108, 301186, 301229, 301299, 301330, and 301378) carry $100.0 \%$ of the R-type alleles in the 16 LAFBRs. When checking the background of these 20 resistant cultivars, we found that the following four were reported to be highly resistant: LAC23 (Yu et al. 1996), IRAT13 (Abamu et al. 1998; Chen et al. 2000), Saber (Campos-Soriano et al. 2013), and C101A51 (Chen et al. 2008; Chen 1996; Mithrasena et al. 2012). These cultivars could be used for the breeding of blast resistance in rice and as genetic materials for gene mapping and cloning.

\section{Conclusions}

Using GWAS, we identified 16 LAFBRs associated with rice blast resistance in the field. Among them, 13 are novel and the other three are co-localized in known blast $\mathrm{R}$ gene regions. The candidate genes in the LAFBRs encode receptor-like protein kinases, transcription factors, and other defense-related proteins. These results suggest that the genetic architecture of resistance against the multiple strains that are typical at field sites differs from that against the single isolate that are typically tested in the greenhouse or growth chamber. Based the rice transcriptome data, we found that seven candidate genes in the 16 LAFBR regions are up-regulated and that five genes are down-regulated in the incompatible interaction.

We also identified 20 rice cultivars in the RDP1 that confer high levels of resistance to $M$. oryzae under both field and growth chamber conditions. These 20 cultivars 
will be useful for the breeding of blast resistance in rice. We also demonstrated that the TRJ sub-population is the most resistant group in the RDP1 and is a potentially useful resource for the breeding of blast resistance.

\section{Methods}

\section{Plant Materials}

The rice RDP1, which contains 413 O. sativa accessions from 82 countries, was provided by the Genetic StocksOryza (GSOR) Collection, USDA ARS Dale Bumpers National Rice Research Center. The RDP1 represents six major sub-populations, including Indica (IND, 87 accessions), Aus (AUS, 57 accessions), Tropical japonica (TRJ, 97 accessions), Temperate japonica (TEJ, 96 accessions), and Aromatic (ARO, 14 accessions) (Zhao et al. 2011). The other 62 accessions have an admixed ancestry, and most are classified as admixtures (ADM) between Temperate and Tropical japonica groups.

\section{Inoculation and Evaluation of Blast Resistance}

The RPD1 cultivars were screened in three hot spots of rice blast in the rice production areas of China: Shanghang (in Southeast China, Fujian Province), Wuchang (in Northeast China, Heilongjiang Province), and Taojiang (in Central China, Hunan Province). The 413 tested rice lines were sown and transplanted in the disease nursery beds with 30 plants/plot; the highly susceptible cultivar Lijiangxintuanheigu (LTH) was sown on the plot borders. A randomized block design with two replications was used for the field screens in the three locations.

The RDP1 cultivars were scored using a 0-9 scale for disease severity according to the Standard Evaluation System of IRRI (1996) (Chaudhary 1996): 0 indicates no infection, i.e., immunity (IM); 1 indicates a highly resistant (HR) reaction, with only very small spots on leaves; 2 indicates a resistant $(\mathrm{R})$ reaction, with small lesions; 3 indicates a moderately resistant (MR) reaction, with small elliptical lesions; 4 indicates a moderately susceptible (MS) reaction, with $<2 \%$ expanding, elliptical lesion area; 5 indicates a susceptible (S) reaction, with a lesion area $<10 \% ; 6$ and 7 indicate susceptible reactions, with a lesion area of $10-25 \%$ and $26-50 \%$, respectively; 8 and 9 indicate highly susceptible (HS) reactions, with a lesion area of $51-75 \%$ and $>75 \%$, respectively.

\section{Construction of the Phylogenetic Tree of the RDP1}

We re-constructed the phylogenetic tree of the RDP1 using 3835 high quality SNP markers selected from the 44-K SNP markers.

\section{GWAS Analysis of the RDP1 Resistance in the Field} The methods used to identify LAFBRs were similar to those previously described (Kang et al. 2016). Tassel 3.0 software and the MLM (mixed linear model) were used in the analysis. We re-estimated the appropriate $K$ value following the previously published method (Evanno et al., 2015) and found that $K=6$ is the best for the RDP1 population. Base on the studies on the rice linkage disequilibrium decay at the $\mathrm{Xa} 5$ locus (100 kb) (Garris et al. 2003) and in different Oryza species (40-500 kb) (Mather et al. 2007), we selected the associated regions using the following standard: $\leq 250$ gwkb with at least three significant SNPs ( $p$-value $\leq 1 \mathrm{E}-5)$.

Bioinformatics Analysis of the LAFBRs in the Rice Genome We obtained the DNA sequences of the LAFBR regions from the reference genome of MSU.V7.0 (http://rice.plantbiology.msu.edu/) and analyzed these sequences using BLAT by aligning all the 56,591 rice gene sequences to the LAFBR regions to obtain candidate genes. We classified the identified genes using a similar method previously described (Kang et al. 2016). First, the genes belonging to known $\mathrm{R}$ gene families were selected as candidate genes. Second, we expanded our search to defense-related genes that encode the following proteins: NBS-LRR, LRR-TMD, kinase, LRR-kinase or CC-TMD, transcription factor, ubiquitin-related E3 ligase, oxidase/oxidoreductase, protein phosphatase and heat shock protein and etc.

\section{RNA-seq Data Used in This Study}

The RNA-seq data sets were downloaded from the ricerice blast interaction sequencing project (GenBank accession number: DRX001418 for compatible rice-rice blast interaction transcriptome and DRX001419 for rice-rice blast incompatible interaction transcriptome).

\section{Additional Files}

Additional file 1: Table S1. Rice blast evaluation of the RDP1 in three blast nurseries in the field. (XLSX $39 \mathrm{~kb})$

Additional file 2: Table S2 Forty rice cultivars that were resistant in all three blast nurseries in the field. (XLSX 14 kb)

Additional file 3: Table S3. Information on the 74 candidate blast resistant genes in LAFBRs. (XLSX $90 \mathrm{~kb}$ )

Additional file 4: Table S4. The 12 strong candidate genes in the LAFBRs that are up-regulated or down-regulated in the rice-rice blast incompatible interaction. (XLSX $10 \mathrm{~kb}$ )

Additional file 5: Table S5. Pair-wise correlation analysis of RDP1 disease reactions evaluated under in the field vs. in the growth chamber. (XLSX $10 \mathrm{~kb}$ )

Additional file 6: Table S6. The SNP haplotypes of the 20 highly resistant cultivars in the 16 LAFBRs. (XLSX $22 \mathrm{~kb}$ )

Acknowledgements

This research was supported by grants from the National Natural Science Foundation of China to H.K. (Grant number 31571964 and 31201476) and from the 973 Project (Grant number 2012CB114005) of the Ministry of Science and Technology of China to G.-L.W. We thank the Genetic Stocks Oryza (GSOR) collection laboratory, the USDA Dale Bumpers National Rice 
Research Center, for providing the seeds of the Rice Diversity Panel 1 cultivars

\section{Authors' Contributions}

DZ: Conducted experiments, performed original data analysis and wrote manuscript; HK: Designed and conducted experiments, analyzed wholegenome sequence data and wrote the manuscript; ZL: Grew rice plants and performed blast inoculations and data analysis; ML and XZ: Grew rice plants; WY, DW and ZW: Increased rice seeds; WL: Wrote and edited the manuscript; GLW: Supervised the experiments and edited the manuscript. All authors read and approved the final manuscript.

\section{Competing Interests}

The authors declare that they have no competing interests.

\section{Author details}

'State Key Laboratory for Biology of Plant Diseases and Insect Pests, Institute of Plant Protection, Chinese Academy of Agricultural Sciences, Beijing 100193, People's Republic of China. ${ }^{2} H u n a n$ Provincial Key Laboratory of Crop Germplasm Innovation and Utilization and College of Agronomy, Hunan Agricultural University, Changsha, Hunan 410128, People's Republic of China. ${ }^{3}$ Department of Plant Pathology, Ohio State University, Columbus, OH 43210, USA.

\section{Received: 14 April 2016 Accepted: 22 August 2016}

\section{Published online: 30 August 2016}

\section{References}

Abamu FJ, Akinsola EA, Alluri K (1998) Applying the AMMI models to understand genotype-by-environment (GE) interactions in rice reaction to blast disease in Africa. Int J Pest Manag 44(4):239-245

Ali ML, Zhao K, Tung C-W, Wright M, Reynolds A, Bustamante C, McClung A, McClung A, Mccouch S, Eizenga G (2010) Screening and association mapping of rice blast disease resistance using a diverse collection of rice germplasm, Symposium Proceedings., pp 12-14

Campos-Soriano L, Valè G, Lupotto E, Segundo BS (2013) Investigation of rice blast development in susceptible and resistant rice cultivars using a gfpexpressing Magnaporthe oryzae isolate. Plant Pathol 62(5):1030-1037

Chaudhary RC (1996) Standard evaluation system for rice. International Rice Research Institute, Manila

Chen DH (1996) Phenotypic Characterization of the Rice Blast Resistance Gene Pi2(t). Plant Dis 80(1):52-56

Chen DH, Nelson RJ, Wang GL, Inukai T, Mackill DJ, Ronald PC (2000) Characterization of Blast Resistance in the Durably Resistant Rice Cultivar Moroberekan. Advances in Rice Blast Research. Springer, Netherlands, pp 17-27

Chen HQ, Chen ZX, Shen NI, Zuo SM, Pan XB, Zhu XD (2008) Pyramiding Three Genes with Resistance to Blast by Marker-Assisted Selection to Improve Rice Blast Resistance of Jin 23B. Chin J Rice Sci 1:005

Copenhaver GP, Famoso AN, Zhao K, Clark RT, Tung C-W, Wright MH, Bustamante C, Kochian LV, McCouch SR (2011) Genetic Architecture of Aluminum Tolerance in Rice (Oryza sativa) Determined through GenomeWide Association Analysis and QTL Mapping. PLoS Genet 7(8):e1002221

Dangl JL, Jones JDG (2001) Plant pathogens and integrated defence responses to infection [J]. Nature 411(6839):826-833

Devi SJSR, Singh K, Umakanth B, Vishalakshi B, Renuka P, Sudhakar KV, Prasad MS, Viraktamath BC, Babu VR, Madhav MS (2015) Development and Identification of Novel Rice Blast Resistant Sources and Their Characterization Using Molecular Markers. Rice Sci 22(5):300-308

Eizenga GC, Ali ML, Bryant RJ, Yeater KM, McClung AM, McCouch SR (2014) Registration of the Rice Diversity Panel 1 for Genomewide Association Studies. J Plant Registrations 8(1):109-116

Eizenga G, McClung A, McClung A, Wright M, Greenberg A, Cobb J, Jung J, Clark R, Singh N, Agosto-Perez F (2013) Using the rice diversity panel 1 to develop novel germplasm for breeding, International Rice Genetics Symposium., pp 161-162

Feng H, Hao Z, Liu J, Wang Z, Wang G, Agronomy CO, University HA (2014) Recent advances in understanding the innate immune mechanisms and developing new disease resistance breeding strategies against the rice blast fungus Magnaporthe oryzae in rice. Yi chuan = Hereditas/Zhongguo yi chuan xue hui bian ji 36(8):756-765
Garris AJ, McCOUCH SR, Kresovich S (2003) Population structure and its effect on haplotype diversity and linkage disequilibrium surrounding the xa5 locus of rice (Oryza sativa L.). Genetics 165(2):759-769

Hu H, Xiong L, Yang Y (2005) Rice SERK1 gene positively regulates somatic embryogenesis of cultured cell and host defense response against fungal infection. Planta 222(1):107-117

Huang X, Wei X, Sang T, Zhao Q, Feng Q, Zhao Y, Li C, Zhu C, Lu T, Zhang Z, Li M, Fan D, Guo Y, Wang A, Wang L, Deng L, Li W, Lu Y, Weng Q, Liu K, Huang T, Zhou T, Jing Y, Li W, Lin Z, Buckler ES, Qian Q, Zhang QF, Li J, Han B (2010) Genome-wide association studies of 14 agronomic traits in rice landraces. Nat Genet 42(11):961-967

Hwang EY, Song Q, Jia G, Specht JE, Hyten DL, Costa J, Cregan PB (2014) A genome-wide association study of seed protein and oil content in soybean. BMC Genomics 15(1):1

Jones JD, Dangl JL (2006) The plant immune system. Nature 444(7117):323-329

Kang H, Wang Y, Peng S, Zhang Y, Xiao Y, Wang D, Qu S, Li Z, Yan S, Wang Z (2016) Dissection of the genetic architecture of rice resistance to the blast fungus Magnaporthe oryzae. Mol Plant Pathol 17(6):959-972

Kawahara Y, Oono Y, Kanamori H, Matsumoto T, Itoh T, Minami E (2012) Simultaneous rna-seq analysis of a mixed transcriptome of rice and blast fungus interaction. Plos One 7(11):e49423

Khush GS (2005) What it will take to feed 5.0 billion rice consumers in 2030. Plant Mol Biol 59(1):1-6

Liebranda TW, van den Berga GC, Zhao Zhadnga PS, Cordewenerb JH, Americab AH, Sklenard J, Jonesd AM, Tamelinga WI, Robatzekd S, Thommaa BP (2013) Receptor-like kinase SOBIR1/EVR interacts with receptor-like proteins in plant immunity against fungal infection. PNAS 110(24):10010-10015

Liu X, Inoue H, Hayashi N, Jiang C-J, Takatsuji H (2015) CC-NBS-LRR-Type R Proteins for Rice Blast Commonly Interact with Specific WRKY Transcription Factors. Plant Mol Biol Report 34(2):533-537

Mather KA, Caicedo AL, Polato NR et al (2007) The extent of linkage disequilibrium in rice (Oryza sativa L.). Genetics 177(4):2223-2232

Meyers BC, Kozik A, Griego A et al (2003) Genome-wide analysis of NBS-LRRencoding genes in Arabidopsis [J]. Plant Cell 15(4):809-834

Mithrasena Y, Wijesundera W, Wijesundera R, Wimalasiri D, Priyanthi R (2012) Pathogenic and Genetic Diversity of Magneporthe oryzae Populations from Sri Lanka. Rice Sci 19(3):241-246

Norton GJ, Douglas A, Lahner B, Yakubova E, Guerinot ML, Pinson SR, Tarpley L, Eizenga GC, McGrath SP, Zhao FJ, Islam MR, Islam S, Duan G, Zhu Y, Salt DE, Meharg AA, Price AH (2014) Genome wide association mapping of grain arsenic, copper, molybdenum and zinc in rice (Oryza sativa L.) grown at four international field sites. PLoS One 9:e89685

Oliveira-Garcia E, Valent B (2015) How eukaryotic filamentous pathogens evade plant recognition. Curr Opin Microbiol 26:92-101

Skamnioti P, Gurr SJ (2009) Against the grain: safeguarding rice from rice blast disease. Trends Biotechnol 27(3):141-150

Spindel J, Begum H, Akdemir D, Collard B, Redoña E, Jannink J, McCouch S (2016) Genome-wide prediction models that incorporate de novo GWAS are a powerful new tool for tropical rice improvement. Heredity 116:395-408

Yu J, Pressoir G, Briggs WH, Bi IV, Yamasaki M, Doebley JF, McMullen MD, Gaut BS, Nielsen DM, Holland JB, Kresovich S, Buckler ES (2006) A unified mixedmodel method for association mapping that accounts for multiple levels of relatedness. Nat Genet 38(2):203-208

Yu ZH, Mackill DJ, Bonman JM, Mccouch SR, Guiderdoni E, Notteghem JL, Tanksley SD (1996) Molecular mapping of genes for resistance to rice blast (Pyricularia grisea Sacc.). TAG Theor Appl Genet Theoretische Und Angewandte Genetik 93(5-6):859-863

Zhao K, Tung CW, Eizenga GC, Wright MH, Ali ML, Price AH, Norton GJ, Islam MR, Reynolds A, Mezey J, McClung AM, Bustamante CD, McCouch SR (2011) Genome-wide association mapping reveals a rich genetic architecture of complex traits in Oryza sativa. Nat Commun 2:467

Zheng W, Yan W, Wang L, Ma Z, Zhao J, Ping W, Zhang L, Liu Z, Lu X (2016) Genetic mapping and molecular marker development for Pi65(t), a novel broad-spectrum resistance gene to rice blast using next-generation sequencing. Theor Appl Genet 129(5):1035-1044 